\title{
CONSENSO ARGENTINO DE CIRUGÍA METABÓLICA
}

\section{ARGENTINE CONSENSUS ON METABOLIC SURGERY}

\author{
Sociedad Argentina de Diabetes (SAD), Sociedad Argentina de Nutrición (SAN), \\ Sociedad Argentina de Cirugía de la Obesidad (SACO)
}

\begin{abstract}
Marianela Aguirre Ackermann', Omar Alva², María Graciela Álvarez ${ }^{3}$, Marisa Andreoni $^{4}$, Eduardo Babor ${ }^{5}$, Verónica Bottino ${ }^{6}$, Oscar Brasesco ${ }^{7}$, Lilia Cafaro ${ }^{8}$, Mónica Coqueugniot $^{9}$, Susana Fuentes ${ }^{10}$, Martín Garaycoechea $^{11}$, Emilio Hidalgo ${ }^{12}$, Adriana Immerso ${ }^{13}$, Ana Iturrospe ${ }^{14}$, Viviana Lasagni ${ }^{15}$, León Litwak ${ }^{16}$, Hugo Montemerlo ${ }^{17}$, Carla Musso ${ }^{18}$, Pablo Omelanczuk ${ }^{19}$, Mariano Palermo ${ }^{20}$, Natalia Pampillón ${ }^{21}$, María del Pilar Quevedo ${ }^{22}$, Clarisa Reynoso ${ }^{23}$, Marcelo Rondina ${ }^{24}$, Graciela Rubin ${ }^{25}$, Magalí Sánchez ${ }^{26}$, Edgardo Serra ${ }^{27}$
\end{abstract}

\section{Coordinadores:}

Etapa prequirúrgica: Susana Fuentes (SAD), Marianela Aguirre Ackermann (SAN), Lilia Cafaro (SACO) Etapa quirúrgica: Eduardo Babor (SAD), Edgardo Serra (SAN), Oscar Brasesco (SACO) Etapa post-quirúrgica: Carla Musso (SAD), Magalí Sánchez (SAN), María Graciela Álvarez (SACO) Secretaría General: María del Pilar Quevedo

\section{RESUMEN}

Introducción: la creciente pandemia de obesidad y diabetes tipo 2 (DM2) demanda opciones terapéuticas más efectivas para lograr un adecuado control metabólico y disminuir la morbimortalidad cardiovascular en este grupo de pacientes. En este contexto, la cirugía metabólica (CM) constituye una herramienta innovadora, segura y eficaz que complementa pero no reemplaza a los cambios necesarios del estilo de vida y tratamiento médico.

Objetivos: el objetivo del Consenso es la fundamentación y acuerdo de utilización de una técnica quirúrgica, específicamente el Bypass Gástrico en Y de Roux (BPGYR), en el tratamiento de pacientes con DM2 que presentan un índice de masa corporal (IMC) entre $30-35 \mathrm{~kg} / \mathrm{m}^{2}$ e inadecuado control metabólico. Conclusiones: el Consenso Argentino de Cirugía Metabólica elaborado por la Sociedad Argentina de Diabetes (SAD), la Sociedad Argentina de Nutrición (SAN) y la Sociedad Argentina de Cirugía de la Obesidad (SACO) expresa la opinión de expertos sobre la evidencia científica disponible y propone considerar a la $\mathrm{CM}$ en el algoritmo terapéutico de pacientes con DM2 e IMC $30-35 \mathrm{~kg} / \mathrm{m}^{2}$ que no logran adecuado control metabólico con tratamiento médico convencional. Se describen los criterios a tener en cuenta en la selección de pacientes para CM, y se destaca el rol del equipo multidisciplinario liderado por médicos especialistas en enfermedades endocrino-metabólicas en la selección, evaluación, preparación y seguimiento de estos pacientes.

Palabras clave: cirugía metabólica, cirugía bariátrica, diabetes mellitus tipo 2, obesidad.

Revista de la Sociedad Argentina de Diabetes 2015; Vol. 49 (95-110)

\section{ABSTRACT}

Introduction: the increasing worldwide pandemic of obesity and type 2 diabetes push us to choose therapeutic options to control metabolic parameters and decrease cardiovascular risk in this group of patients. Metabolic surgery (MS) became an innovative, efficient and safe tool complementing rather than replacing lifestyle changes and pharmacologic therapy.

Objectives: the aim of the consensus was the rationale and agreement to use a surgical technique, specifically the Roux bypass surgery (BPGYR), in patients with DM2 with a BMI between $30-35 \mathrm{~kg} / \mathrm{m}^{2}$ and inadequate metabolic parameters.

Conclusions: the first Argentinean metabolic surgery consensus was done by the Argentine Diabetes Society (SAD), Argentine Nutrition Society (SAN) and Argentine Society of Obesity Surgery (SACO) and the proposal was to consider metabolic surgery in type 2 diabetic patients with BMI between $30-35 \mathrm{~kg} /$ $\mathrm{m}^{2}$ after been treated with the best medical therapy.

We describe selection criteria of patients, the important role of the interdisciplinary approach, evaluation, and preparation before surgery and the follow up.

Key words: metabolic surgery, bariatric surgery, type 2 diabetes mellitus, obesity.

Revista de la Sociedad Argentina de Diabetes 2015; Vol. 49 (95-110) 
1 Médica Especialista en Clínica Médica y Nutrición. Magister en Diabetes. Equipo de Cirugía Bariátrica y Metabólica, Centro CIEN. Corrientes-Misiones

2 Médico Especialista en Psiquiatría. Equipo de Cirugía Bariátrica y Metabólica, Centro CIEN-DIAGNOMED. Hospital Argerich. CETAL. CABA

3 Médica Especialista en Clínica Médica y Nutrición. Centro de Estudio y Tratamiento de la Obesidad Severa (CETOS). CABA

4 Lic. en Nutrición. Obesidad y Cirugía Mini Invasiva (OCMI). CABA

5 Médico Cirujano. CETOS. CABA

6 Lic. en Nutrición. OCMI. CABA

7 Médico Cirujano. Director de OCMI. Hospital Austral. Fundación Favaloro. Sanatorio Finochietto. SACO. CABA

8 Médica Especialista en Nutrición. OCMI. CABA

9 Lic. en Nutrición. CETOS. CABA

10 Médica Especialista en Clínica Médica y Diabetes. Equipo de Cirugía Bariátrica y Metabólica, Hospital de Alta Complejidad El Cruce. Florencio Varela, Buenos Aires

11 Médico Cirujano. Equipo de Cirugía Bariátrica y Metabólica. Hospital de Alta Complejidad El Cruce. Florencio Varela, Buenos Aires

12 Médico Especialista en Psiquiatría. Equipo de Cirugía Bariátrica y Metabólica, Centro CIEN. Corrientes-Misiones

13 Lic. en Nutrición. OCMI. CABA

14 Médica Especialista en Nutrición y Diabetes. OCMI. CABA

15 Lic. en Psicología. Diplomada en Psicología Bariátrica. Clínica Quirúrgica de la Obesidad (CQO). Mendoza

16 Médico Especialista en Endocrinología. Hospital Italiano de Buenos Aires. Sociedad Argentina de Diabetes. CABA

17 Médico Especialista en Nutrición. Sanatorio Mater Dei, Unidad de Soporte Nutricional. Sociedad Argentina de Nutrición. CABA
18 Médica Especialista en Endocrinología. Fundación Favaloro. CABA

19 Médico Cirujano. Hospital Italiano de Mendoza. Centro Quirúrgico de la Obesidad. Mendoza

20 Médico Cirujano. MAAC. FACS. Equipo de Cirugía Bariátrica y Metabólica. Centro CIEN-DIAGNOMED. CABA

21 Lic. en Nutrición. Centro Quirúrgico de la Obesidad. Mendoza

22 Médica Especialista en Clínica Médica y Nutrición. Equipo de Cirugía Bariátrica y Metabólica. Centro CIEN-DIAGNOMED. CABA

${ }^{23}$ Lic. en Nutrición. Hospital Británico de Buenos Aires. CABA

24 Médico Cirujano. Equipo de Cirugía Bariátrica y Metabólica. Hospital de Alta Complejidad El Cruce, Florencio Varela, Buenos Aires

${ }_{25}$ Médica Especializada en Diabetes. Hospital Privado Universitario de Córdoba S.A. Córdoba

${ }^{26}$ Médica Especialista en Clínica Médica. Centro Quirúrgico de la Obesidad. Mendoza

27 Médico Cirujano. Equipo de Cirugía Bariátrica y Metabólica, Centro CIEN, Corrientes-Misiones. CIEN-DIAGNOMED. CABA

Contacto del autor: María del Pilar Quevedo

E-mail: maria.pquevedo@gmail.com

Correspondencia: Viamonte 2146 piso 50 "B" (C1056ABH), CABA, Argentina

Fecha de trabajo recibido: 13/10/15

Fecha de trabajo aceptado: 10/11/15

Conflictos de interés: los autores declaran que no existen conflicto de interés 


\section{INTRODUCCIÓN}

En 2014 un grupo multidisciplinario de representantes de la Sociedad Argentina de Diabetes (SAD), la Sociedad Argentina de Nutrición (SAN) y la Sociedad Argentina de Cirugía de la Obesidad (SACO) se reunió para elaborar el I Consenso Argentino de Cirugía Metabólica. El objetivo de este Consenso es la fundamentación y acuerdo de utilización de una técnica quirúrgica, específicamente el Bypass Gástrico en Y de Roux (BPGYR), en el tratamiento de pacientes con diabetes mellitus tipo 2 (DM2) que presentan un índice de masa corporal (IMC) entre $30-35 \mathrm{~kg} / \mathrm{m}^{2}$ e inadecuado control metabólico.

\section{METODOLOGÍA}

Este Consenso se basó en una revisión y síntesis de la evidencia científica publicada junto con la evaluación y experiencia de los médicos especialistas en Endocrinología y Diabetes, Clínica Médica, Nutrición, Cirugía, Psiquiatría, Lic. en Nutrición y Psicología designados por la SAD, SAN y SACO. Los resultados expresan la opinión de expertos (nivel de evidencia C) luego de analizar la evidencia científica disponible. El Consenso fue aprobado por los Comités Científicos de SAN, SAD y SACO en abril de 2015. Se reevaluarán sus conclusiones cada dos años.

\section{RESULTADOS}

\section{Cirugía metabólica}

Se denomina cirugía metabólica (CM) a la intervención que se realiza sobre el tubo digestivo con el objetivo de conseguir la mejoría o remisión de la DM2 y la reducción del riesgo cardiometabólico ${ }^{1,2,3}$.

$\mathrm{La} \mathrm{CM}$ es un proceso que comprende la preparación prequirúrgica, el acto quirúrgico y el seguimiento post-quirúrgico en el que se requiere la participación activa de especialistas con profundo conocimiento y experiencia de las disciplinas intervinientes.

En el año 1991, el National Institute of Health (NIH) definió los criterios de indicación de cirugía bariátrica (CB) basados en el IMC y comorbilidades de riesgo ${ }^{4}$. Desde entonces, hubo una evolución de los conocimientos científicos vinculados al tratamiento de la obesidad, DM2 y enfermedades asociadas. En 2007 se realizó en Roma la primera Reunión Cumbre en Cirugía de la Diabetes, donde un grupo de expertos elaboró las directrices sobre $\mathrm{CM}$, considerándola una opción terapéutica para el tratamiento de la $\mathrm{DM} 2^{5}$.
La American Diabetes Association (ADA) en Standard of Medical Care 2009 incluye por primera vez a la CB como opción terapéutica en pacientes obesos con DM2 e inadecuado control metabólico con tratamiento médico convencional ${ }^{6}$.

La International Diabetes Federation (IDF), en su Position Statement 2011, establece criterios de elegibilidad de CB en pacientes con DM2 e IMC $>30 \mathrm{~kg} / \mathrm{m}^{2}$. En el subgrupo de pacientes con DM2 e IMC entre 30 y $35 \mathrm{~kg} / \mathrm{m}^{2}$ considera a la CB como un tratamiento alternativo cuando la DM2 no puede ser adecuadamente controlada con tratamiento médico óptimo, especialmente en presencia de otro factor de riesgo cardiovascular mayor. Además propone que la CB debería incluirse en los algoritmos diseñados para el tratamiento de la DM2?.

Las Guías AACE/TOS/ASMBS $2013^{8}$ y EASO/ IFSO-EC ${ }^{9}$ recomiendan la $\mathrm{CB}$ para el tratamiento de la DM2 en pacientes con inadecuado control metabólico e IMC $>35 \mathrm{~kg} / \mathrm{m}^{2}$. También proponen que en pacientes con IMC $30-35 \mathrm{~kg} / \mathrm{m}^{2}$ cuidadosamente seleccionados, la CB podría considerarse una opción terapéutica.

Sin embargo, para la CM el IMC podría no ser un criterio apropiado de selección, ya que no refleja la composición corporal ni la distribución de la masa grasa ${ }^{1,3,5,10,11,12,13,14,15}$. Por lo tanto, resulta imprescindible definir criterios adecuados, más allá del IMC, que reflejen el perfil metabólico de los pacientes para su apropiada selección.

\section{Selección del paciente}

En numerosas publicaciones se han discutido las indicaciones de $\mathrm{CM}$, sin contar hasta el momento con un consenso definitivo ${ }^{16,17,18}$. En este Consenso hemos definido cuáles deberían ser los criterios de inclusión para la selección de los pacientes candidatos para $\mathrm{CM}^{7,17}$.

Para la indicación quirúrgica, los pacientes deberán reunir los criterios de inclusión más básicos:

- Dos o más criterios mayores y/o;

- Un criterio mayor más dos criterios menores.

- Criterios de inclusión básicos:

- DM2 $\geq 2$ años de evolución.

- Edad $\leq 65$ años, salvo condición especial que lo haga recomendable.

- HbA1C >8\% durante al menos un año con fracaso al tratamiento médico adecuado. Se define tratamiento médico adecuado a la combinación de triple terapia farmacológica utilizando metformina, sulfonilureas, inhibidores de DPP-4, agonis- 
tas GLP-1, tiazolidinedionas, inhibidores SGLT2 e insulina de acuerdo a los estándares de cuidado médico para la DM2, dirigido por especialistas en enfermedades endocrino-metabólicas.

- Indicación quirúrgica realizada por médico especialista en enfermedades endocrino-metabólicas (especialistas en Endocrinología, Nutrición, Diabetología, etc.), en forma conjunta con equipo quirúrgico debidamente constituido y entrenado en $\mathrm{CM}$.

- IMC 30-34,9 kg/m² 17 .

- Circunferencia de cintura en hombres >102 $\mathrm{cm}$ y en mujeres $>88 \mathrm{~cm}$.

- Péptido $\mathrm{C}$ en ayunas $>1 \mathrm{ng} / \mathrm{dl}^{9,17}$.

- Compromiso y adherencia a la preparación y posibilidad de seguimiento con el equipo interdisciplinario. El equipo evaluará el perfil psicológico del paciente y la accesibilidad para llevar a cabo los requisitos necesarios (consultas, suplementación, etc.).

- Criterios mayores:

- Hipertensión arterial (definida por tensión arterial sistólica sostenida $>139 \mathrm{mmHg}$ o tensión arterial diastólica sostenida $>89 \mathrm{mmHg}$ ), con o sin tratamiento.

- Dislipidemia, con o sin tratamiento, definida por7:

a. Colesterol total $>200 \mathrm{mg} / \mathrm{dl}$ y/o;

b. Colesterol LDL >100 mg/dl y/o;

c. Triglicéridos $>150 \mathrm{mg} / \mathrm{dl}$ y/o;

d. Colesterol no-HDL $>130 \mathrm{mg} / \mathrm{dl}$.

- Antecedentes personales de enfermedad macro y/o microvascular.

- Síndrome de apnea hipopnea obstructiva de sueño (SAHOS) moderada-severa7,19.

- Criterios menores:

- Hiperinsulinemia de ayuno definida por insulinemia en ayunas mayor de $20 \mathrm{mcU} / \mathrm{ml}$ (en pacientes sin tratamiento con insulina exógena).

- Espesor de la capa íntima media carotidea $\geq 1 \mathrm{~mm}$.

- Historia familiar de eventos cardiovasculares -infarto agudo de miocardio (IAM), accidente cerebrovascular (ACV)- en familiares de primer grado (padre $<55$ años y/o madre $<65$ años).

- Enfermedad grasa del hígado no alcohólica (EGHNA) diagnosticada por enzimas hepáticas elevadas y/o métodos de diagnóstico por imágenes.

- Criterios de exclusión:

- Autoinmunidad positiva definida por anticuerpos antiGAD.

- Fragilidad clínica (según escala Edmonton).

-Trastornos psiquiátricos (ver Anexo 2).
- Historia de adicciones y abuso de sustancias.

La evaluación clínica, nutricional y psicológica realizada por los integrantes del equipo interdisciplinario es similar a la realizada a los pacientes con DM2 sometidos a CB8,20,21 (Anexos 1 y 2).

\section{Definición de resolución (remisión y recurrencia) de la DM2}

Se considerarán como referencia los conceptos de remisión definidos por el grupo de expertos de ADA de $2009^{6}$ y los criterios de recurrencia propuestos por Brethauer et al. en el año $2013^{22}$.

- Remisión: glucemias menores al nivel de diagnóstico de diabetes en ausencia de terapia farmacológica o quirúrgica (por ejemplo, dispositivos endoluminales $)^{6,23}$. La remisión puede ser parcial o completa:

- Remisión parcial: hiperglucemia menor al nivel de diagnóstico de diabetes ( $\mathrm{HbA} 1 \mathrm{C}<6,5 \%$ y glucemias de ayuno 100-125 mg/dl) durante al menos un año y en ausencia de terapia farmacológica.

- Remisión completa: implica el retorno a valores de glucemia normales ( $\mathrm{HbA} 1 \mathrm{C}<6 \%$ ) durante un año y en ausencia de terapia farmacológica.

- Remisión prolongada: remisión completa que dura más de cinco años.

- Recurrencia: glucemia o $\mathrm{HbA} 1 \mathrm{C}$ en rango de diabetes (glucemia de ayuno $\geq 126 \mathrm{mg} \%$ y $\mathrm{HbA} 1 \mathrm{C} \geq 6,5 \%$ ) o necesidad de medicación para la diabetes después de haber tenido remisión parcial o completa ${ }^{22}$.

\section{Rol de las distintas técnicas quirúrgicas en la resolución de la DM2}

Durante los últimos 10 años numerosas publicaciones demostraron mejoría en el control glucémico y disminución de la mortalidad y eventos cardiovasculares después de $\mathrm{CB} / \mathrm{CM}^{22,43,44,45}$. El BPGYR fue la primera cirugía en probar su eficacia en remisión de DM2 en pacientes con obesidad mórbida. El BPGYR por vía laparoscópica es la técnica más aceptada, de mayor seguridad y eficacia, y tiene una mayor tasa de remisión a largo plazo de DM2 que otros procedimientos restrictivos por lo que es considerada la cirugía gold standard ${ }^{24}$.

- Descripción de la técnica (BPGYR): haciendo referencia sólo a los aspectos técnicos que tienen impacto en los resultados y en la disminución de complicaciones, describimos:

- Pouch gástrico: volumen aproximado de 30 a $50 \mathrm{ml}$, calibrado con una sonda de 34-36 Fr. Se 
debe aislar completamente el fondo gástrico. El tamaño aproximado es de $4 \mathrm{~cm} \times 7 \mathrm{~cm}$. Anastomosis gastroentérica: se aconseja con técnica mixta mecánica-manual, aunque puede realizarse en forma completamente manual. Siempre debe ser calibrada con una sonda de 34-36Fr. Asa biliar: se aconseja una longitud de $100 \mathrm{~cm}$ desde el ángulo de Treitz. Asa alimentaria: se recomienda una longitud de $150 \mathrm{~cm}$ desde la anastomosis gastroentérica. Anastomosis enteroentérica: se sugiere la confección con técnica mixta mecánica manual. Ascenso de las asas: se recomienda el ascenso antecólico-antegástrico con el fin de disminuir la posibilidad de obstrucción intestinal. Brechas mesentéricas: es aconsejable el cierre de las brechas del espacio de Pettersen y del espacio intermesentérico; esto disminuye la incidencia de hernias internas. Pruebas de hermeticidad: se recomienda la realización de la prueba de azul de metileno al finalizar la intervención. Drenajes: se sugiere dejar un drenaje en la cavidad abdominal durante siete días aproximadamente ${ }^{25}$.

\section{Mecanismos de remisión de la DM2}

Diversos factores han sido descriptos como determinantes en la remisión de la DM2 luego del BPGYR $^{26,27}$. Los cambios en la homeostasis glucémica se deben tanto a mecanismos relacionados al descenso de peso secundario, a la restricción calórica como a otros mecanismos independientes que son propios del BPGYR ${ }^{28}$.

Se describen dos factores que contribuyen a la mejoría del control glucémico en forma inmediata a la cirugía: el aumento de la sensibilidad hepática a la insulina y la mejoría en la función de la célula $\beta$ asociada a la secreción postprandial de GLP-129,30,31,32,33. Al progresar la pérdida de peso, aumenta aún más la sensibilidad periférica a la insulina ${ }^{34}$. Paralelamente, el aumento de la secreción de GLP1 colabora en la mejoría de la tolerancia a la glucosa, aunque no sería el único factor causal $35,36,37$.

Otros mecanismos tales como cambios en la recirculación y concentración de ácidos biliares ${ }^{38}$, modificaciones en la microbiota intestinal ${ }^{39}$, remodelamiento intestinal ${ }^{40}$ y gluconeogénesis intestinal ${ }^{41}$, cambios en el sensado intestinal de nutrientes $^{42}$ y efectos de las citoquinas inflamatorias, podrían contribuir a la mejoría del metabolismo de la glucosa post BGYR.

\section{Resultados}

a) Morbimortalidad de pacientes con DM2 sometidos a cirugía electiva

Diversos estudios observacionales -prospectivos y retrospectivos- han destacado la reducción en la mortalidad del $30-40 \%$ en sujetos con obesidad mórbida en tratamiento quirúrgico versus tratamiento médico convencional ${ }^{46,47,48,49,50}$.

La CB en los pacientes obesos con DM2 tiene múltiples beneficios para la salud, incluyendo la reducción de la mortalidad por todas las causas ${ }^{47}$. Sin embargo, hasta el momento son pocos los estudios que evaluaron el efecto de la CM sobre la mortalidad específicamente en pacientes diabéticos, por lo que se requeriría mayor número de pacientes seguidos a largo plazo.

b) Eficacia: resultados (comparación con tratamiento médico de la DM2)

La comparación del mejor tratamiento médico versus tratamiento quirúrgico en el control metabólico ha demostrado mejores resultados en la rama quirúrgica $51,52,53,54,55,56,57,58,59$. Estudios randomizados controlados mostraron una remisión de DM2 22 veces mayor (RR 22.1 -IC 3.2-154.3; $P=0,002-)$ con tratamiento quirúrgico versus tratamiento convencional $52,54,56,57,60,61$. El estudio prospectivo no randomizado SOS mostró remisión de DM2 con tratamiento quirúrgico del $72 \%$ a dos años y del $36 \%$ a 10 años $^{62}$. A pesar de la declinación en la remisión de la diabetes a los 10 años se mantuvo una reducción significativa de eventos macrovasculares ${ }^{63}$. Con respecto a la evolución de la enfermedad microvascular, los estudios disponibles demuestran estabilidad o mejoría de la misma ${ }^{64,65}$.

\section{Seguridad}

\section{a) Complicaciones quirúrgicas}

Las complicaciones quirúrgicas no difieren de las observadas en pacientes obesos con BPGYR. La mortalidad quirúrgica es 0,28-0,35\% (menor que el $0,35-0,60 \%$ de la mortalidad asociada con colecistectomía laparoscópica) 1,66,67.

Ngiam y Lee describen una tasa de mortalidad general del $0,02 \%$ en los estudios evaluados en pacientes con IMC $<35 \mathrm{~kg} / \mathrm{m}^{2}$ y una incidencia de complicaciones quirúrgicas mayores y menores del $8,7 \%{ }^{68}$. Cohen et al. no refieren complicaciones quirúrgicas mayores dentro de los 30 días post-cirugía ni mortalidad en un grupo de 66 pacientes con DM2 e IMC $30-35 \mathrm{~kg} / \mathrm{m}^{2}$. Sin em- 
bargo revelan $15 \%$ de complicaciones menores como hematomas en la pared abdominal, úlcera de la anastomosis e infección urinaria. Las complicaciones mayores publicadas son filtraciones anastomóticas, hernias internas, estenosis de la neoboca, hemorragias, trombosis venosa profunda, tromboembolismo, reintubación traqueal, drenajes percutáneos, reoperaciones abdominales y aquellas que requieran reinternación. Estas complicaciones se informan en menor medida en pacientes con IMC $<35 \mathrm{~kg} / \mathrm{m}^{2}$ que en pacientes con IMC mayor ${ }^{14}$.

\section{b) Complicaciones nutricionales y metabólicas} luego de cirugía de la DM2

Las complicaciones metabólicas y nutricionales podrían ocurrir como consecuencia del cambio en la absorción de macro y micronutrientes o bien por una insuficiente adherencia a la suplementación vitamínico-mineral8,20,21,69,70,71 (Tabla 1).

\begin{tabular}{|l|l|}
\hline Complicaciones nutricionales & Complicaciones nutricionales \\
\hline Anemia & Hiperoxaluria y nefrolitiasis \\
\hline $\begin{array}{l}\text { Alteración en el metabolismo } \\
\text { mineral óseo }\end{array}$ & Síndrome de dumping \\
\hline Hipocalcemia & Hipoglucemia \\
\hline Hipovitaminosis D & Nesidioblastosis \\
\hline Hiperparatiroidismo secundario & \\
\hline
\end{tabular}

Tabla 1: Complicaciones nutricionales y metabólicas.

\section{Costo-efectividad}

Análisis recientes revelan la eficacia de la CB en la reducción del uso de fármacos para el tratamiento de DM2 y enfermedades asociadas, como así también en los costos de cuidado sanitario en pacientes con DM2 que podrían extrapolarse a la $\mathrm{CM}$. Diversos estudios señalan que el BPGYR es costo-efectivo para DM2 e IMC 30-35 kg/m² 72,73,74. Los resultados del análisis de sensibilidad sugieren que, sobre todo en el horizonte temporal de 20 años, el tratamiento es costo-efectivo.

\section{Seguimiento post-quirúrgico del paciente}

Aunque se trata de pacientes con obesidad grado I, previo a la cirugía no hay evidencia de pérdida excesiva de peso luego de la $\mathrm{CM}^{3,11}$.

El monitoreo de rutina clínico-endocrinológiconutricional se recomienda después de todos los procedimientos de CB (Grado A) $)^{8,21}$, y en CM res- peta los lineamientos propuestos para BPGYR.

En CB está ampliamente aceptada la necesidad de suplementación de micronutrientes en forma empírica durante toda la vida70 (Anexo 1).

\section{Grado de adherencia a las pautas}

Algunas publicaciones indican baja adherencia a las recomendaciones alimentarias de pacientes post $\mathrm{CB}^{75}$, no existiendo datos sobre adherencia luego de $\mathrm{CM}$ específicamente. Los profesionales deben remarcar la importancia de la adherencia a la suplementación vitamínica y mineral durante toda la vida del paciente para prevenir carencias nutricionales ${ }^{76}$.

\section{Recurrencia de DM2. Manejo farmacológico de la recurrencia}

a) Enfoque terapéutico

EI BPGYR se asocia con remisión duradera de DM2 en gran número de sujetos con obesidad severa, aunque un tercio de ellos podría experimentar recaída dentro de los primeros cinco años de la remisión inicial ${ }^{77}$.

A pesar de la recurrencia, el $75 \%$ de los pacientes con DM2 mantiene un buen control metabólico con $\mathrm{HbA} 1 \mathrm{C}<7 \% 22$ y el $90 \%$ no requiere insulina ${ }^{56}$. Algunos estudios con seguimiento a largo plazo señalan que los pacientes con remisión prolongada post $\mathrm{CB}$ o con recaída pero que conservan adecuado control metabólico tienen menor desarrollo de enfermedad macrovascular y microvascular, y mantendrían el "efecto legado" $47,7,78,79$. Faltan estudios para determinar la evolución de enfermedad micro y macrovascular luego de la recaída de la DM277,22.

El $80-100 \%$ de los pacientes debe suspender o disminuir la medicación para DM2 en el post operatorio inmediato; por este motivo en pacientes en tratamiento previo con insulina el monitoreo debe ser más estricto ${ }^{77}$.

La medicación debe elegirse de acuerdo al impacto sobre el peso. Los fármacos recomendados por ser neutros o beneficiosos con respecto al peso corporal son: metformina, incretinas (análogos GLP1 e inhibidores DPP4) e inhibidores SGLT2.

\section{CONCLUSIONES}

La creciente pandemia de obesidad y DM2 demanda opciones terapéuticas efectivas. Lograr el adecuado control metabólico y disminuir la morbimortalidad cardiovascular continúa siendo un desafío en este grupo de pacientes. En este 
contexto, la CM constituye una herramienta innovadora, segura y eficaz que complementa pero no reemplaza a los cambios necesarios del estilo de vida y tratamiento médico.

Este Consenso propone considerar a la CM en el algoritmo terapéutico de pacientes con DM2 e IMC 30-35 kg/m² e inadecuado control metabólico con tratamiento médico convencional. Se destaca el rol del equipo multidisciplinario liderado por médicos especialistas en enfermedades endocrinometabólicas en la selección, evaluación, preparación y seguimiento de estos pacientes.

\section{REFERENCIAS}

1. Rubino F, Cummings DE. The coming age of metabolic surgery. Nat. Rev. Endocrinol. 2012; 8:702-704.

2. Fruhbeck G. Bariatric and metabolic surgery: a shift in eligibility and success criteria. Nat. Rev. Endocrinol. 2015;11:465-477.

3. Shimizu H, Timratana P, Schauer PR, et al. Review of metabolic surgery for type 2 diabetes in patients with a $\mathrm{BMI}<35 \mathrm{~kg} / \mathrm{m}^{2}$. Journal of Obesity 2012.

4. National Institutes of Health Consensus Development Panel. Gastrointestinal surgery for severe obesity. Consensus Development Conference Statement. Ann. Intern. Med. 1991; 115:956-61.

5. Rubino F, Kaplan LM, Schauer PR, Cummings DE. The diabetes surgery summit consensus conference: recommendations for the evaluation and use of the gastrointestinal surgery to treat type 2 diabetes mellitus. Ann. Surg. 2012; 215 (3):339- 405.

6. American Diabetes Association. Standards of Medical Care in Diabetes-2009. Diabetes Care 2009; 32: S13-S61.

7. Dixon JB, Zimmet P, Rubino F. Bariatric surgical and procedural interventions in the treatment of obese patients with type 2 diabetes. A position statement from the International Diabetes Federation Taskforce on Epidemiology and Prevention. Surg. Obes. Relat. Dis. 2011; 7: 433-47.

8. Mechanick JI, Youdim A, Jones DB. Clinical Practice Guidelines for the perioperative nutritional, metabolic, and nonsurgical support of the bariatric surgery patient-2013 update: cosponsored by American Association of Clinical Endocrinologists. The Obesity Society and American Society for Metabolic and Bariatric Surgery. Endocrine Practice 2013; 19:1-36.

9. Fried M, Yumuk V, Oppert JM, et al. Interdisciplinary European Guidelines on Metabolic and Bariatric Surgery. Obes. Surg. 2014; 24:42-55.

10. Pories WJ, Dohm LG, Mansfield CJ. Beyond the BMI: the search for better guidelines for bariatric surgery. Obesity 2010;18: 865871.

11. Cohen R. Pinheiro JC, Schiavon C, Salles JE. Effects of gastric bypass surgery in patients with type 2 diabetes and only mild obesity. Diabetes Care 2012; 35: 1420-1428.

12. Sjoholm K, Anvenden A, Peltonen M, et al. Evaluation of current eligibility criteria for bariatric surgery: diabetes prevention and risk factor changes in Swedish obese subjects (SOS) study. Diabetes Care 2013; 365:1335-40.

13. Rubino F, Sula A, Moreira M, et al. Bariatric, metabolic and diabetes surgery: what's in a name? Ann. Surg. 2014;259:117-122.

14. Cohen R, Caravatto PP, Petry T. Metabolic surgery for type 2 diabetes in patients with a BMI of $<35 \mathrm{~kg} / \mathrm{m}^{2}$ : a surgeon's perspective. Obes. Surg. 2013; 23: 809-818.
15. Schulman AP, del Genio F, Sinha N, Rubino F. Metabolic surgery for treatment of type 2 diabetes mellitus. Endocr. Pract. 2009;15: 624-631.

16. Ramos-Leví AM, Rubio Herrera MA. Metabolic surgery: quo vadis? Endocrinol. Nutr. 2014; 61: 35-46.

17. Cohen R. Metabolic surgery: who and when? Is there a good answer. Nutr. Hosp. 2013; 28:14-16.

18. Neff KJ, le Roux CW. Bariatric surgery: the indications in metabolic disease. Dig. Surg. 2014; 31:6-12.

19. Nogueira F, y col. Guías prácticas de diagnóstico y tratamiento del síndrome de apneas e hipopneas obstructivas del sueño. Revista Medicina 2013; 73.

20. Mechanick JI, Kushner RF, Sugerman HJ. American Association of Clinical Endocrinologists, The Obesity Society and the American Society for Metabolic and Bariatric Surgery Medical Guidelines for clinical practice for the perioperative nutritional, metabolic and non surgical support of the bariatric patient. Endocr. Practice 2008; 14: 1-83.

21. Pampillon N. Consenso Argentino de Nutrición en Cirugía Bariátrica. Actualización en Nutrición 2011; 12:98-141.

22. Brethauer SA, Schauer PR. Can diabetes be surgically cured? Long-term metabolic effects of bariatric surgery in obese patients with type 2 diabetes mellitus. Ann. Surg. 2013; 258:628-36.

23. Buse JB, Caprio S, Cefalu WT, et al. How do we define cure of diabetes? Diabetes Care 2009; 32:2133-2135

24. Helmio M, Victorzon M, Ovaska J, et al. SLEEVEPASS: a randomized prospective multicenter study comparing laparoscopic sleeve gastrectomy and gastric bypass in the treatment of morbid obesity: preliminary results. Surg. Endosc. 2012; 26:2521-2526.

25. Cohen R, Schiavon C, Pinheiro J, et al. Duodeno-jejunal bypass for the treatment of type 2 diabetes in patients with body mass index of 22-34 kg/m²: a report of 2 cases. Surg. Obes. 2007; 3:195-197.

26. Elahi D, Galiatsatos $P$, Rabiee A, et al. Mechanisms of type 2 diabetes resolution after Roux-en-Y gastric bypass. Surgery for Obesity and Related Diseases 2014; 10: 1028-1040.

27. Rubino F. Is type 2 diabetes an operable intestinal disease? Diabetes Care 2008; 31:S290-296.

28. Seeley R, Chambers A, Sandoval D. The role of gut adaptation in the potent effects of multiple bariatric surgerie on obesity and diabetes. Cell Metab 2015; 3: 369-378.

29. Madsbad S, Dirksen C, Holst J. Mechanisms of changes in glucose metabolism and bodyweight after bariatric surgery. Lancet Diabetes Endocrinol. 2014; 2: 152-64.

30. Dutia R, Brakoniecki K, Bunker P, et al. Limited recovery of b-cell function after gastric bypass despite clinical diabetes remission. Diabetes 2014; 63:1214-1223.

31. Young Min Cho. A gut feeling to cure diabetes: potential mechanisms of diabetes remission after bariatric surgery. Diabetes Metab. J. 2014; 38:406-415.

32. Weiss $R$, Ram Weiss. Effects of Roux-en-Y gastric bypass on bcell function Diabetes 2014; 63:1171-1173.

33. Rubino F, Amiel AA. Is the gut the "sweet spot" for the treatment of diabetes? Diabetes 2014; 63:2225-2228.

34. Bojsen-Möller K, Dirksen C, Jörgensen N, et al. Early enhancements of hepatic and later of peripheral insulin sensitivity combined with increased postprandial insulin secretion contribute to improved glycemic control after Roux-en-Y gastric bypass. Diabetes 2014;63:1725-1737.

35. Manning S, Pucci A, Batterham R. GLP-1: a mediator of the beneficial metabolic effects of bariatric surgery? Physiology 2015; 30: 50-62. 
36. Nannipieri M, Baldi S, Mari A, et al. Roux-en-Y gastric bypass and sleeve gastrectomy: mechanisms of diabetes remission and role of gut hormones. J. Clin. Endocrinol. Metab. 2013; 98: 43914399.

37. Kashyap SR, Daud S, Kelly KR, et al. Acute effects of gastric bypass versus gastric restrictive surgery on b-cell function and insulinotropic hormones in severely obese patients with type 2 diabetes. International Journal of Obesity (2009) 1-10.

38. Gerhard G, Styer A, Wood C, et al. A role for fibroblast growth factor 19 and bile acids in diabetes remission after Roux-en-Y gastric bypass. Diabetes Care 2013; 36: 1859-1864.

39. Sweeney T, Morton J. Metabolic surgery: action via hormonal milieu changes, changes in bile acids or gut microbiota? A summary of the literature. Best Practice \& Research Clinical Gastroenterology 2014; 28: 727-740.

40. Saedi N. Control in rats after gastric bypass reprogramming of intestinal glucose metabolism and glycemic. Science 2013; 341: 406-410.

41. Troy S, Sooty M, Ribeiro M, et al. Intestinal gluconeogenesis is a key factor for early metabolic shanges after gastric bypass but not after gastric lap-band in mice. Cell Metab. 2004; 3: 201-211.

42. ZietekT, Daniel H. Intestinal nutrient sensing and blood glucose control. Curr. Opin. Clin. Nutr. Metab. Care 2015, 18:381-388.

43. Pories WJ, Swanson MS, MacDonald KG, et al. Who would have thought it? An operation proves to be the most effective therapy for the adult- onset diabetes mellitus. Ann. Surg. 1995;222:339-50.

44. Schauer PR, Burguera B, Ikramuddin S, et al. Effect of iaparoscopic Roux-enY gastric bypass on type 2 diabetes mellitus. Ann. Surg. 2003;238:467-84.

45. AdamsTD, Davidson LE, Litwin SE, et al. Health benefits of gastric bypass surgery after 6 years. JAMA 2012; 308:1122-31.

46. Adams TD, Gress RE, Smith SC, et al. Long-term mortality after gastric bypass surgery. N. Engl. J. Med. 2007; 357: 753-61.

47. Sjöström L, Narbro K, Sjöström CD, et al. Effects of bariatric surgery on mortality in Swedish obese subjects. N. Engl. J. Med. 2007; 357: 741-52.

48. Christou NV, Sampalis JS, Liberman M, et al. Surgery decreases long-term mortality, morbidity, and health care use in morbidly obese patients. Ann. Surg. 2004; 240: 416-24.

49. Pontiroli $A E$, Morabito $A$. Long-term prevention of mortality in morbid obesity through bariatric surgery a systematic review and meta-analysis of trials performed with gastric banding and gastric bypass. Ann. Surg. 2011; 253: 484-7.

50. Flum DR. Impact of gastric bypass operation on survival: a population-based analysis. J. Am. Coll. Surg. 2004; 199:543-51.

51. Dixon JB, le Roux CW, Rubino F, Zimmet P. Bariatric surgery for type 2 diabetes. Lancet 2012; 379: 2300-11.

52. Mingrone G, Panunzi S, De Gaetano A, et al. Bariatric surgery versus conventional medical therapy for type 2 diabetes. $\mathrm{N}$. Engl. J. Med. 2012; 366:1577-1585.

53. Kashyap SR, Bhatt DL, Wolski K, et al. Metabolic effects of bariatric surgery in patients with moderate obesity and type 2diabetes: analysis of a randomized control trial comparing surgery with intensive medical treatment. Diabetes Care 2013; 36: 2175-82.

54. Schauer PR, Kashyap SR, Wolski K, et al. Bariatric surgery versus intensive medical therapy in obese patients with diabetes. $\mathrm{N}$. Engl. J. Med. 2012; 366:1567-76.

55. Kashyap SR, Bhatt DL, Schauer PR. Bariatric surgery vs. advanced practice medical management in the treatment of type 2 diabetes mellitus: rationale and design of the Surgical Therapy and Medications Potentially Eradicate Diabetes Efficiently trial (STAMPEDE). Diabetes Obes. Metab. 2010; 12:452-4.
56. Schauer PR, Bhatt DL, Kirwan J, et al. Bariatric surgery versus intensive medical therapy for diabetes 3-year outcomes. N. Engl. J. Med. 2014

57. Ikramuddin S, Korner J, Lee WJ, et al. Roux-en-Y gastric bypass vs intensive medical management for the control of type 2 diabetes, hypertension, and hyperlipidemia: the Diabetes Surgery Study Randomized Clinical Trial. JAMA 2013; 309: 2240-49.

58. Courcoulas AP, Goodpaster BH, Eagleton JK, et al. Surgical vs medical treatments for type 2 diabetes mellitus: a randomized clinical trial. JAMA Surg 2014; published online 4 Jun.

59. Halperin F, Ding SA, Simonson DC, et al. Roux-en-Y gastric bypass surgery or lifestyle with intensive medical management in patients with type 2 diabetes: feasibility and 1-year results of a randomized clinical trial. JAMA Surg 2014; published online 4 Jun.

60. Gloy VL, Briel M. Bariatric surgery versus non surgical treatment for obesity: a systematic review for weight loss. BMJ 2013; 347: f5934. Doi: 10.1136/bmj.f5934.

61. Dixon JB, O' Brien PE, Playfair J, et al. Adjustable gastric banding and conventional therapy for type 2 diabetes: a randomized controlled trial. JAMA 2008; 299: 316-23.

62. Sjöström L, Lindroos AK, Peltonen M, et al. Lifestyle, diabetes, and cardiovascular risk factors 10 years after bariatric surgery. N. Engl. J. Med. 2004; 351:2683-93.

63. Sjöström L. Association of bariatric surgery with long term remission of type 2 diabetes and with microvascular and macrovascular complications. JAMA 2014; 311:2297-304.

64. Miras A, le Roux C. Metabolic surgery: shifting the focus from glycaemia and weight to end-organ health. Lancet Diabetes Endocrinol. 2014; 2: 141-51.

65. Carlsson LMS, Romeo S, Jacobson P, et al. The incidence of albuminuria after bariatric surgery and usual care in Swedish obese subjects (SOS): a prospective controlled intervention trial. International J. Obes. 2015; 39:169-175.

66. Buchwald H, Estok R, Fahrbach $\mathrm{K}$, et al. Trends in mortality in bariatric surgery: a systematic review and meta-analysis. Surgery, 2007; 142: 621-635.

67. Khuri SF, Najjar SF, Daley J, et al. Comparison of surgical outcomes between teaching and nonteaching hospitals in the Department of Veterans Affairs. Annals of Surgery 2001; 234:370383.

68. Ngiam KY, Lee WJ, Lee YC, et al. Efficacy of metabolic surgery on $\mathrm{HbA} 1 \mathrm{c}$ decrease in type 2 diabetes mellitus patients with $\mathrm{BMI}$ $<35$ kg/m². A review. Obes. Surg. 2014; 24:148-158.

69. Kellene A. Nutrition and metabolic support recommendations for the bariatric patient. Nutr. Clin. Pr 2014; 29:6: 718-739.

70. Amaya García MJ. Micronutrientes en cirugía bariátrica. Nutrición Hospitalaria 2012; 27:349-361.

71. Savino P, Carvajal C, Nassar R, et al. Necesidades nutricionales específicas después de cirugía bariátrica. Rev. Colomb. Cir. 2013; 28:161-171.

72. Ikramuddin S, Klingman D, Swan T, et al. Cost-effectiveness of Roux-en-Y gastric bypasses in type 2 diabetes patients. Am. J. Manag. Care. 2009 Sep; 15(9):607-15.

73. Keating CL, Dixon JB, Moodie ML, et al. Cost-efficacy of surgically induced weight loss for the management of type 2 diabetes: a randomized controlled trial. Diabetes Care. 2009 Apr; 32(4):580-4.

74. Picot J, Jones J, Colquitt JL, et al. Weight loss surgery for mild to moderate obesity: a systematic review and economic evaluation. Obes. Surg. 2012 Sep; 22(9):1496-506.

75. Moize V, Geliebter A, Gluck ME, et al. Obese patients have inadequate protein intake related to protein intolerance up to 1 year following Roux-en-Y gastric bypass. Obes. Surg. 2003; 13:23-8. 
Revista de la Sociedad Argentina de Diabetes Vol. 49 No 3 Diciembre de 2015: 95-110 ISSN 0325-5247 / ISSN $2346-9420$ (en línea)

76. Welch G, Wesolowski C, Zagarins S, et al. Evaluation of clinical outcomes for gastric bypass surgery: results from a comprehensive follow-up study. Obes. Surg. 2011; 21: 18-28.

77. Arterburn DE, Bogart A, Sherwood N, et al. A multisite study of long-term remission and relapse of type 2 diabetes mellitus following gastric bypass. Obes. Surg. 2013; 23: 93-102.
78. Sjöström L, Peltonen $\mathrm{M}$, Jacobson $\mathrm{P}$, et al. Bariatric surgery and long-term cardiovascular events. JAMA 2012; 307: 56-65.

79. Romeo S, Maglio C, Burza MA. Cardiovascular events after bariatric surgery in obese subjects with type 2 diabetes. Diabetes Care 2012; 35:2613-2617. 


\section{ANEXO I}

\section{Evaluación y control del paciente diabético}

Se debe realizar una evaluación médica completa para evaluar el grado de control de la diabetes, posibles complicaciones y detección de factores de riesgo asociados ${ }^{1}$. La tasa de remisión de la DM2 post CB está relacionada con el tipo de diabetes, por lo cual es importante su evaluación (Tabla 1).

\begin{tabular}{|l|l|}
\hline Historia médica & $\begin{array}{l}\text { Edad y características de inicio de la diabetes } \\
\text { Historia de la enfermedad y tratamientos anteriores recibidos } \\
\text { Episodios de complicaciones agudas de la diabetes } \\
\text { Tratamiento actual de la diabetes, incluyendo tratamiento higiénico-dietético, medicación y comorbilidades } \\
\text { asociadas, adherencia y resultados del automonitoreo } \\
\text { Historia de complicaciones crónicas relacionadas a la DM: } \\
\text {. Microvascular: retinopatía, nefropatía, neuropatía (sensitiva, motora, autonómica, incluyendo disfunción } \\
\text { sexual y gastroparesia) } \\
\text {. Macrovascular: enfermedad cardiovascular, enfermedad cerebrovascular y enfermedad vascular periférica } \\
\text {. Otras: problemas psicosociales, enfermedad periodontal, etc. }\end{array}$ \\
\hline Examen físico & $\begin{array}{l}\text { Peso, talla, IMC, circunferencia de cintura } \\
\text { Frecuencia cardíaca. TA, incluyendo investigación de hipotensión ortostática cuando esté indicado }\end{array}$ \\
$\begin{array}{l}\text { Fondo de ojo } \\
\text { Examen de la piel } \\
\text { Examen del pie }\end{array}$ \\
$\begin{array}{l}\text { No difiere del solicitado de rutina para BPGYR, con énfasis en: } \\
\text { G Glucemia en ayunas } \\
\text {. HbA1C } \\
\text {. Péptido C en ayunas } \\
\text {. Perfil lipídico } \\
\text {. Hepatograma } \\
\text {. Albuminuria mediante albúmina/creatinina en muestra aislada de orina } \\
\text {. Creatinina sérica y MDRD } \\
\text {. Autoanticuerpos (ej. anti GAD) }\end{array}$ \\
\hline
\end{tabular}

Tabla 1: Evaluación prequirúrgica del paciente con DM2.

\section{Control glucémico}

Se debe optimizar el control de la glucemia preoperatoria utilizando un plan de atención integral de la diabetes, incluyendo plan alimentario, actividad física y tratamiento médico (Grado A, nivel 1$)^{22}$.

Los targets para control glucémico preoperatorio deben ser: $\mathrm{HbA} 1 \mathrm{c} \leq 7 \%$, glucemia en ayunas $\leq 110 \mathrm{mg} / \mathrm{dl}$, glucemia postprandial $\leq 140 \mathrm{mg} / \mathrm{dl}$ (Grado $\mathrm{A}$, nivel 1$)^{22}$. Targets más amplios como HbA1C de 7 a $8 \%$ deben ser considerados en pacientes con complicaciones macro o microvasculares avanzadas o condiciones comórbidas extensas, o diabetes de larga evolución en la cual el objetivo ha sido difícil de alcanzar (Grado A, nivel 1$)^{22}$.

En pacientes con $\mathrm{HbA} 1 \mathrm{C}>8 \%$ o diabetes no controlada, el juicio clínico determinará la oportunidad de la cirugía (Grado D) ${ }^{22}$.

\section{Preparación nutricional prequirúrgica}

En todos los pacientes con DM2 se recomienda la terapia nutricional como un componente eficaz en el tratamiento de la enfermedad independientemente de la intervención quirúrgica'. En $\mathrm{CM}$ las recomendaciones nutricionales son similares a las de CB, y los principales objetivos son el descenso de peso previo a la cirugía, la reducción del tejido adiposo visceral y volumen hepático, y la corrección de las alteraciones metabólicas para prevenir complicaciones, entre otras $2,3,4,5,6,7$.

Estudios han demostrado que un descenso de peso del 5-10\% resulta en una efectiva reducción de la glucemia en ayunas y $\mathrm{HbA} 1 \mathrm{C}$ comparables con los objetivos logrados con la farmacoterapia, por lo cual el descenso preoperatorio podría usarse para lograr los objetivos glucémicos en estos pacientes $^{8}$. Además, junto con la mejoría glucémica, la disminución del 10\% del exceso de peso previa a la cirugía mejora parámetros bioquímicos de inflamación². Más aún, existe consenso en la literatura que la pérdida de peso preoperatoria se correlaciona con una disminución del tamaño hepático y de la grasa intraabdominal ${ }^{9}$, facilitando el acto quirúrgico 
al mejorar la exposición de los campos operatorios, acortar el tiempo quirúrgico y disminuir la pérdida sanguínea10,11,12,13,14, así como el número de complicaciones a corto plazo post-operatorio ${ }^{15}$.

\section{Composición nutricional}

La Asociación Americana de Diabetes (ADA) y la Asociación Europea para el Estudio de la Diabetes (EASD) establecen recomendaciones de cantidades y tipos de macronutrientes ${ }^{1,16}$. Con respecto al valor calórico, en DM2 un plan hipocalórico que produzca un descenso de peso moderado $(5$ a $9 \mathrm{~kg}$ ) ha demostrado incrementar la sensibilidad de la insulina y disminuir la hiperglucemia, las dislipemias y la HTA. Se recomienda una distribución del $50 \%$ de hidratos de carbono con un mínimo de 130- $150 \mathrm{~g}$ para evitar la cetosis; 0,8 a 1,0 g/ $\mathrm{kg}$ peso ideal/día de proteínas con un máximo del $20 \%$ del valor calórico total y $30 \%$ de grasas con selección, disminuyendo las saturadas a $\leq 7 \%$ del total de calorías (Grado A), aumentando los ácidos grasos monoinsaturados y poliinsaturados, y reduciendo el consumo de colesterol 1,7,17,18,19. Según el Consenso Argentino de Nutrición en Cirugía Bariátrica se aconsejan $10 \mathrm{~g} /$ día de fibra soluble. La recomendación para la población general de reducir el sodio a $2.300 \mathrm{mg} /$ día también es apropiada para las personas con diabetes (Grado B) 1 .

Para acelerar la disminución del volumen hepático, en las dos semanas previas a la cirugía, se puede utilizar una dieta de muy bajas calorías (DMBC) -800 Kcal diarias aproximadamente- con un mínimo de $150 \mathrm{~g}$ de hidratos, $25 \%$ del valor calórico total compuesto por proteínas de alto valor biológico, entre 7 y 10 g/día de grasas seleccionadas para asegurar la ingesta de ácidos grasos esenciales y $10 \mathrm{~g}$ de fibra soluble ${ }^{3,6,18,20,21}$. Se debe aportar suplementación vitamínico-mineral para cubrir el $100 \%$ del requerimiento diario. En caso de existir déficit, debe realizarse además la reposición antes de la cirugía ${ }^{17}$.

\section{Seguimiento post-quirúrgico del paciente}

El monitoreo de rutina metabólico y nutricional se recomienda después de todos los procedimientos de CB (Grado A) ${ }^{22}$ y en CM respeta los lineamientos propuestos para BPGYR.

\section{3.a. Seguimiento nutricional}

El objetivo principal es optimizar la mejoría metabólica con remisión total o parcial de la DM2 y de las comorbilidades asociadas ${ }^{22}$. Entre los objetivos secundarios se destacan principalmente: 1) disminución del 50\% del exceso de peso 17,23,24,25,26,27 no existiendo estudios que evidencien una pérdida de peso excesivo después de la realización de procedimientos quirúrgicos convencionales en pacientes con IMC $30-35 \mathrm{~kg} / \mathrm{m}^{2} 28$; 2) mantenimiento del peso corporal, pues la reganancia de peso podría asociarse a recurrencia de la DM2 ${ }^{24,25}$.

El esquema de frecuencia de consultas no difiere del propuesto en CB: primera consulta a la semana de la cirugía, segunda consulta a los 14 días, tercera consulta al mes de la cirugía. Desde el segundo mes y durante todo el primer año post-cirugía, una consulta por mes. Durante el segundo año posterior a la cirugía, cada tres meses (15, 18, 21 y 24 meses). Luego, como mínimo, las consultas deben ser bianuales según el grado de adhesión que haya presentado el paciente con respecto a las pautas de alimentación y actividad física indicadas, a la patología asociada y al resultado obtenido en la remisión de la DM2 ${ }^{17}$.

La dieta post $\mathrm{CM}$ no difiere de la propuesta para BPGYR de CB, debiendo satisfacer las cantidades mínimas diarias recomendadas de nutrientes y calorías para lograr buena cicatrización y preservar la masa magra durante el período de descenso rápido de peso, con volumen reducido y consistencia adecuada para mantener una buena tolerancia ${ }^{17}$. La alimentación se progresa por etapas iniciando con consistencia líquida, volumen disminuido y alto fraccionamiento hasta llegar a consistencia sólida con seis ingestas diarias (Grado D) 17,22,29.

El valor energético al inicio no supera las 1.000 kcal/día y aumenta paulatinamente a 1.200-1.400 kcal/día a partir de los 6 a 12 meses, según tolerancia.

La ingesta proteica debe ser de al menos $60 \mathrm{~g} /$ día y hasta $1,5 \mathrm{~g} / \mathrm{kg} / \mathrm{peso}$ ideal de proteínas de alto valor biológico (Grado D) 17,22,29.

Se recomienda un aporte mínimo de 150 g/día de HC complejos. Los simples deben evitarse o limitarse por el aporte de calorías que dificultaría la pérdida de peso y por la posibilidad de producir síndrome de Dumping (Grado D) ${ }^{17,22}$.

Las grasas no deberán superar del 25 al 30\% del valor calórico total diario, con selección adecuada para cubrir requerimientos de ácidos grasos esenciales ${ }^{17}$. Se recomienda aumentar el aporte de los ácidos grasos de cadena larga n-3 (EPA y DHA) y n-3 alfa linolénico (ALA) (Grado B)'.

Se utiliza fibra soluble durante los primeros 
meses ya que la insoluble puede ser mal tolerada por la hipoclorhidria que no permite la adecuada disgregación de las paredes celulares. Incluso algunos tipos de fibra no digeribles pueden generar un pequeño bezoar o atascar la salida del pouch ${ }^{17}$.

La recomendación de sodio es la misma que para la población en general, menos de 2.300 mg/ día y adecuar en forma individual en caso de hipertensión (Grado B) 1 .

Se debe asegurar la ingesta de al menos 1,5 I/ día de líquidos, iniciando de a pequeños sorbos, utilizando bebidas sin gas, sin calorías ni cafeína (Grado D) $)^{1722}$.

El consumo de alcohol puede aumentar el riesgo de hipoglucemia en pacientes diabéticos ${ }^{10}$. Debido a la importancia de la enzima alcohol deshidrogenasa de la pared gástrica en el metabolismo del etanol, luego del BPGYR, aumenta el riesgo de una intoxicación aguda o de desarrollar adicción. Se recomienda, por lo tanto, limitar el consumo de alcohol en los pacientes sometidos a cirugía bariátrica ${ }^{17}$.

La progresión debe ser guiada por el nutricionista del equipo (Grado A) ${ }^{22}$. Se deben evitar las preparaciones de alta osmolaridad y los azúcares concentrados para minimizar los síntomas de síndrome de Dumping (Grado D) 17,22,29. En caso de síndrome de Dumping tardío por hipoglucemia el paciente debe ser evaluado y asesorado en consecuencia ${ }^{29}$.

Si bien la evidencia sugiere que no hay un porcentaje ideal de calorías de hidratos de carbono, proteínas y grasas para todas las personas con diabetes, se debe tener en cuenta el requerimiento proteico, el volumen del pouch y la menor absorción de nutrientes de los pacientes sometidos a cirugía bariátrica.

En aquellos pacientes sin resolución completa de su DM2, dislipidemia o hipertensión, la vigilancia continua debe seguir las guías de práctica clínica actuales para su condición (Grado D) ${ }^{22}$.

\section{3. b. Seguimiento clínico-endocrinológico-farma- cológico}

Los resultados del tratamiento quirúrgico de la diabetes tienen relación directa con la adherencia del paciente a las pautas, por lo que el seguimiento deberá plantearse a largo plazo. A través de la vigilancia clínica nutricional se deben prevenir deficiencias nutricionales así como también ajustar el tratamiento de las comorbilidades. Los requerimientos de vitaminas y micronutrientes deberían ser prescriptos en forma rutinaria para compensar la ingesta y absorción reducida.

En caso de utilizar medicación cuando se inicie la vía oral, deben usarse las formas masticables o líquidas, en lugar de la medicación de liberación prolongada para maximizar la absorción (Grado D) ${ }^{22}$.

\section{- Post-operatorio temprano}

En el seguimiento del paciente diabético, en el post-operatorio temprano (menos de cinco días) ${ }^{3}$ deberá implementarse un protocolo de control glucémico estricto durante la internación con determinación de glucemias en ayunas en forma periódica (Grado A) ${ }^{22}$. Se aconsejan determinaciones por glucómetro preprandial, 2 hs. postprandial y bedtime en el domicilio, dependiendo del nivel de control glucémico, el uso de agentes orales o insulina, o si el paciente presenta hipoglucemias (Grado A) ${ }^{22}$.

Se debe discontinuar el uso de drogas secretagogas (sulfonilureas, meglitinidas) y ajustar dosis de insulina para minimizar el riesgo de hipoglucemia (Grado D). Si la DM2 se encuentra en remisión luego de la cirugía se debe retirar la medicación antidiabética (Grado D) ${ }^{22}$.

En pacientes al alta o ambulatorios con hiperglucemia deben considerarse metformina o terapias basadas en incretinas (Grado D) ${ }^{22}$. La metformina puede ser continuada hasta alcanzar resolución prolongada de la diabetes demostrada por normalización de objetivos glucémicos (incluyendo glucemia en ayunas, postprandial y $\mathrm{HbA} 1 \mathrm{C})^{22}$.

\section{- Post-operatorio tardío}

Se recomienda luego de todo procedimiento de cirugía bariátrica/metabólica monitoreo metabólico y nutricional de rutina (Grado A) ${ }^{22}$. La intervención deberá incluir un enfoque interdisciplinario con cambios dietarios, actividad física, modificación de la conducta con seguimiento frecuente, y si es apropiado, terapia farmacológica y/o revisión quirúrgica (Grado B) ${ }^{22}$. La frecuencia de consultas nutricionales no difiere del seguimiento post-quirúrgico de $\mathrm{CB}$.

El seguimiento en el post-operatorio tardío (más de cinco días) ${ }^{3}$ dependerá de la presencia de complicaciones no específicas (cardiovasculares) o específicas (microvasculares) de la diabetes, así como del tiempo de remisión de la misma. En aquellos pacientes con o sin resolución completa de su diabetes, la vigilancia continua y el manejo deben seguir las guías de práctica clínica actuales para esta condición (Grado D) $)^{22}$. 
Los pacientes con BPGYR que presenten síntomas de hipoglucemia postprandial y que no responden a la manipulación nutricional deben someterse a una evaluación para diferenciar hipoglucemia pancreatógena no insulinoma, de causas iatrogénicas o facticias, síndrome de Dumping e insulinoma (Grado C) ${ }^{22}$.

Cuando la remisión completa es mayor a cinco años, deben considerarse metas para pacientes sin diabetes, tanto tiempo como si el paciente permanezca sin recurrencia de diabetes y sin eventos cardiovasculares ${ }^{30}$.

En aquellos pacientes con o sin resolución completa de su dislipidemia o HTA, la vigilancia continua y el tratamiento deben guiarse por las guías de práctica actual para esa condición (Grado D) ${ }^{22}$.

En pacientes con enfermedad grasa no alcohólica del hígado, las transaminasas elevadas deben controlarse periódicamente hasta que alcancen valores normales o estables ${ }^{31}$.

Las drogas antiinflamatorias no esteroideas deben evitarse después de la cirugía, si es posible, por el riesgo de ulceraciones/perforaciones en la anastomosis (Grado D) ${ }^{22}$.

Se debe aconsejar a los pacientes la incorporación de actividad física aeróbica moderada, que incluya un mínimo de 150 minutos con un objetivo de 300 minutos por semana, y ejercicios de fuerza muscular dos a tres veces por semana (Grado A) ${ }^{22}$.

\section{Suplementación de micronutrientes}

Está ampliamente aceptada la necesidad de suplementación de micronutrientes de forma empírica durante toda la vida tras la cirugía bariátri$\mathrm{ca}^{32}$. La recomendación actual de suplementación se describe en la Tabla 22,29,32,33.

\begin{tabular}{|c|c|}
\hline $\begin{array}{l}\text { Multivitamínico } \\
\text { (MVM) }\end{array}$ & Dos comprimidos/día \\
\hline Calcio- citrato & 1.200-1.500 mg/día con 400-800 UI Vit D3 \\
\hline Vitamina D & 3.000 Ul/día \\
\hline B12 & Oral: 500-2.000 ug/día - SL 500 ug/día - IM 1.000 ug/mes ó 3.000 ug cada seis meses \\
\hline Hierro & 45-60 mg/día en MMV \\
\hline Cinc & 15 mg/día en MVM \\
\hline Biotina & 2,5 mg/día en MVM \\
\hline Cobre & 2 mg/día en MVM \\
\hline Ácido fólico & 400 ug/día en MVM \\
\hline Tiamina & 20-30 mg/d en MVM \\
\hline A & $10.000 \mathrm{U} / \mathrm{d}$ en $\mathrm{MVM}$ \\
\hline $\mathbf{E}$ & $15 \mathrm{mg} / \mathrm{d}$ en MVM \\
\hline K & 120 ug/d en MVM \\
\hline
\end{tabular}

Tabla 2: Suplementación preventiva de vitaminas y minerales.

Se recomienda monitoreo basal, seis meses post-cirugía y luego una vez por año de: calcio, PTHi, fósforo, magnesio, $25(\mathrm{OH})$ vitamina $\mathrm{D}$, vitamina B12, ácido fólico, ferritina, \%saturación de transferrina, hemograma, coagulograma y RIN para detectar posibles deficiencias, en cuyo caso se adecuará la suplementación hasta lograr la repleción ${ }^{17,22,29}$. El seguimiento nutricional es fundamental para lograr los objetivos propuestos ${ }^{17,29,32}$ 


\section{REFERENCIAS ANEXO I}

1. American Diabetes Association. Standards of Medical Care in Diabetes-2014. Diabetes Care 2014; 37: S14-S80.

2. Tarnoff M, Kaplan L, Shicora S. An evidenced-based assesment of preoperative weight loss in bariatric surgery. Obes. Surg. 2008; 18:1059-1061.

3. Mechanick JI, Kushner RF, Sugerman HJ. American Association of Clinical Endocrinologists, The Obesity Society and the American Society for Metabolic and Bariatric Surgery Medical Guidelines for clinical practice for the perioperative nutritional, metabolic and non surgical support of the bariatric patient. Endocr. Practice 2008;14: 1-83.

4. Apovian C, Cummings S, Anderson W, et al. Best practice updates for multidisciplinary care in weight loss surgery. Obesity 2009;17:871-79.

5. Kalarchian M, Marcus M. Preoperative weight loss in bariatric surgery. Letter to Editor. Obes. Surg. 2009; 19: 539.

6. Rubio A, Moreno C. Implicaciones nutricionales de la cirugía bariátrica sobre el tracto gastrointestinal. Nutr. Hosp. 2007; 22: 124-34.

7. Torresani ME, Somoza MI. Cuidado nutricional cardiometabólico. Buenos Aires, AKADIA 2011; 8:620-622-648.

8. Youdim A. Reducción del riesgo quirúrgico. Bariatric. Times 2011; 8-9.

9. Martínez-Ramos D, Salvador-Sanchis D, Escrig-Sos J. Pérdida de peso preoperatoria en pacientes candidatos a cirugía bariátrica. Recomendaciones basadas en la evidencia. Cir. Esp. 2012; 90 (3): 147-155.

10. Huerta S, Dredar S, Hayden E, et al. Preoperative weight loss decreases the operative time of gastric bypass at a Veterans Administration Hospital. Obes. Surg. 2008; 18:508-12.

11. Liu RC, Sabnis AA, Forsyth C, et al. The effects of acute preoperative weight loss on laparoscopic Roux-en-Y gastric bypass. Obes. Surg. 2005; 15:1396-402.

12. Alami RS, Morton JM, Schuster R, et al. Is there a benefit to preoperative weight loss in gastric bypass patients. A prospective randomized trial. Surg. Obes. Relat. Dis. 2007; 3:141-6.

13. Edholm D, Kullberg J, Haenni A, et al. Preoperative 4-week lowcalorie diet reduces liver volume and intrahepatic fat, and facilitates laparoscopic gastric bypass in morbidly obese. Obes. Surg. 2011; 21:345-50.

14. Ali MR, Baucom-Pro S, Broderick-Villa GA, et al. Weight loss before gastric bypass: feasibility and effect on postoperative weight loss and weight loss maintenance. SurgObesRelat Dis. 2007; 3:515-20.

15. Van Nieuwenhove $Y$, Dambrauskas Z, Campillo-Soto A, et al. Preoperative very low-calorie diet and operative outcome after laparoscopic gastric bypass. A randomized multicenter study. Arch. Surg. Vol. 146 ( $\left.N^{\circ} 11\right)$, nov. 2011.

16. American Diabetes Association. Standards of Medical Care in Diabetes-2010. Position Statement. Diabetes Care 2011; 34: S11S61.

17. Pampillón N, Reynoso C, Pagano C, et al. Consenso Argentino de Nutrición en Cirugía Bariátrica. Actualización en Nutrición 2011; 12:98-141.
18. Rubio $\mathrm{M}$, Moreno $\mathrm{C}$. Dietas de muy bajo contenido calórico: adaptación a nuevas recomendaciones. Rev. Esp. Obes. 2004; 2:91-9.

19. Martí M, Bragagnolo J. Diabetes tipo 2. Manual de tratamiento. Rosario: Corpus 223-224.

20. Huerta S, Li Z, Anthony T, et al. Feasibility of a supervised inpatient low-calorie diet progrem for massive weight loss prior to RYGB in superobese patients. Obes. Surg. 2010; 20:173-180.

21. Robert J. Preoperative low energy diet diminishes liver size. Obes. Surg. 2004; 14:1165-1170.

22. Mechanick JI, Youdim A, Jones DB. Clinical Practice Guidelines for the perioperative nutritional, metabolic, and nonsurgical support of the bariatric surgery patient-2013 update: cosponsored by American Association of Clinical Endocrinologists, The Obesity Society and American Society for Metabolic and Bariatric Surgery. Endocrine Practice 2013; 19:1-36.

23. Ramos-Leví AM, Rubio Herrera MA. Endocrinol. Nutr.2014; 61:35-46

24. Jimenez A, Casamitjana R, Flores L. Long term effects of seleeve gastrectomy and Roux-en-Y gastric bypass surgery on type 2 diabetes mellitus in morbidly obese subjects. Ann. Surg. 2012; 256:1023-1029.

25. Brethauer $\mathrm{S}$, Aminian $\mathrm{A}$, Romero-Talamas $\mathrm{H}$. Can diabetes be surgically cured? Long-term metabolic effects of bariatric surgery in obese patients with type 2 diabetes mellitus. Ann. Surg. 2013; 258:628-636.

26. Dixon JB, O'Brien PE. Adjustable gastric banding and conventionaltherapy for type 2 diabetes: a randomized controlled trial. JAMA 2008; 299:316-339.

27. Cohen R, Pinheiro JC, Schiavon CA. Effects of gastric bypass surgery in patients with type 2 diabetes and only mild obesity. Diabetes Care 2012; 35:1420-1428.

28. Rubino F. The diabetes surgery Summit Consensus Conference. Recommendations for the evaluation and use of gastrointestinal surgery to treat type 2 diabetes mellitus. Annals of Surgery 2010; 251: 399-405.

29. Fried M, Yumuk V, Oppert JM. International Federation for Surgery of Obesity and Metabolic Disorders-European Chapter (IFSO-EC), European Association for the Study of Obesity Management Task Force (EASO OMTF). Interdisciplinary European Guidelines on Metabolic and Bariatric Surgery. Obes. Surg 2014; 24:42-55.

30. Buse J, Caprio S, Cefalu W. How do we define cure of diabetes? Diabetes Care 2009; 32:2133-2135.

31. Heber D, Greenway FL, Kaplan LM. Endocrine and nutritional management of the post-bariatric surgery patient: an Endocrine Society Clinical Practice Guideline. J. Clin. Endocrinol. Metab. 2010; 95:4823-4843.

32. Amaya García MJ. Micronutrientes en cirugía bariátrica. Nutrición Hospitalaria 2012; 27:349-361.

33. Savino P, Carvajal C, Nassar R, Zundel N. Necesidades nutricionales específicas después de cirugía bariátrica. Rev. Colomb. Cir. 2013; 28:161-171. 


\section{ANEXO II \\ Salud mental}

Lic. Lasagni V, Dr. Alva Lilué O, Dr. Hidalgo E

\section{Introducción}

En relación al tratamiento quirúrgico de la diabetes no existen estudios previos que determinen diferencias en los criterios de valoración, tratamiento y seguimiento para cirugía metabólica o bariátrica, motivo por el cual este apartado respetará los indicadores establecidos por guías internacionales vigentes para la cirugía bariátrica.

\section{Rol de los profesionales de salud mental dentro del equipo quirúrgico}

La cirugía bariátrica es una intervención quirúrgica que pretende que las personas con obesidad disminuyan su peso y no vuelvan a recuperarlo. Esto implica un proceso de preparación, valoración, intervención y seguimiento donde se requiere del trabajo de un equipo interdisciplinario y dentro de equipo, el psicólogo y/o psiquiatra intervienen en las distintas fases del tratamiento.

El rol del profesional de la salud mental consistirá, durante la primera fase pre-quirúrgica, en valorar los aspectos psicológicos del candidato a cirugía bariátrica con el fin de determinar si la persona se encuentra en condiciones de iniciar el tratamiento quirúrgico de acuerdo a los criterios de selección establecidos por las guías nacionales y/o internacionales para tal fin.

En caso de no reunir con las condiciones psicológicas establecidas por las mismas, el profesional de la salud mental deberá indicar su correspondiente tratamiento ya sea para optimizar los recursos internos del paciente, estabilizar su estado psicológico, etc., o en caso de no ser posible el tratamiento quirúrgico indicar su tratamiento correspondiente.

Además será necesario informar aspectos generales del tratamiento quirúrgico tanto al paciente como a sus familiares o responsables y orientar a la persona sobre los pasos de la cirugía bariátrica. Facilitar la adaptación a un nuevo estilo de vida, imagen corporal, hábitos alimentarios, etc. será fundamental para la adherencia al tratamiento para lo cual será necesario coordinar grupos de apoyo terapéutico pre y post-quirúrgico.

\section{Etapa prequirúrgica: valoración de la salud mental}

Es preciso encuadrar la valoración de modo de que la persona comprenda las causas que determinan una demora o limitación de la cirugía y que el rol del psicólogo y psiquiatra será asegurar que la persona llegue en las mejores condiciones a la práctica quirúrgica o prevenir un riesgo mayor. En caso de no ser favorable esta valoración, el profesional psicólogo o psiquiatra deberán determinar otras alternativas de tratamiento.

A partir de los criterios de selección de Rubio $M$ et al., 2004', al igual que los de otros grupos ${ }^{3,4}$ que trabajan con pacientes con obesidad grave que aspiran a una cirugía bariátrica, se formulan las siguientes recomendaciones:

- Estabilidad psicológica:

- Ausencia de abuso de alcohol u otras sustancias psicoactivas.

- Ausencia de otras alteraciones psiquiátricas mayores (psicosis, episodio maníaco, hipomaníaco, mixto o depresivo), retraso mental y bulimia nerviosa. Es muy importante realizar una detallada evaluación de la conducta alimentaria por la importancia que la misma tiene en la evolución psicológica y en los resultados de la cirugía ${ }^{6,7,8}$.

- Tener muy en cuenta la presencia o antecedentes de ideación de muerte y/o suicida por el aumento de la mortalidad por suicidio luego de la cirugía ${ }^{2,5}$.

- Capacidad para comprender los mecanismos por los que se pierde peso con la cirugía y entender que no siempre se alcanzan buenos resultados.

- Comprender que el objetivo de la cirugía no es alcanzar el peso ideal.

- Compromiso de adhesión a las normas de seguimiento tras la cirugía.

Existen, no obstante, situaciones que en razón de su beneficio-riesgo se deben plantear individualmente en el contexto del equipo multidisciplinar.

Estos criterios de selección serán entonces los que determinarán el rol del equipo de salud mental en esta primera fase de valoración prequirúrgica.

El psicólogo y/o psiquiatra deberán valorar al postulante a cirugía de acuerdo a los criterios de selección y enriquecer la valoración interdisciplinaria con distintos indicadores que se aportarán. 


\section{Etapa post-quirúrgica: seguimiento psicológico}

Revisando la distinta bibliografía sobre las técnicas de seguimiento post-operatorio, se observó que existen distintos autores que manifiestan que el seguimiento psicológico puede ser individual y/o grupal.

El rol de psicólogo y/o psiquiatra en esta etapa será fundamentalmente acompañar y favorecer la adaptación de la persona a nuevos estilos de vida o trabajar en la resolución de posibles conflictos. Este objetivo se podrá cumplimentar ya sea a través de tratamientos psicoterapéuticos y/o psiquiátricos de ser necesario, y/o a través de grupos de apoyo y seguimiento psicológico. El grupo de apoyo representa un soporte de suma importancia para hacer la transición hacia una vida distinta.

\section{REFERENCIAS ANEXO II}

1. Rubio M, Martínez C, Vidal O, et al. Documento de Consenso sobre Cirugía Bariátrica. Rev. Esp. Obes. 2004; 4: 223-249.

2. Peterhänsel C, Petroff D. Obesity treatment. Risk of completed suicide after bariatric surgery: a systematic review. Obesity $\mathrm{Re}-$ views 2013; 14: 369-38.

3. Hornero JP, Gastañaduy, Tilve MJ. Valoración psicológica y psiquiátrica de los candidatos a cirugía bariátrica. Papeles del psicólogo 2005 (abril), Nº 90.

4. Zimmerman M, Francione WC, Chelminski I, et al. Presurgical psychiatric evaluations of candidates for bariatric surgery, part 1: reliability and reasons for and frequency of exclusion. J. Clin. Psychiatry 2007; 68: 1557-1562.

5. Tindle HA, Omalu B, Courcoulas A, Marcus M, Hammers J, KuIler LH. Risk of suicide after long term follow up from bariatric surgery. Am. J. Med. 2010; 123: 1036-1042.

6. Alger-Mayer S, Rosati C, Polimeni JM, Malone M. Preoperative binge eating status and gastric bypass surgery: a long term outcome study. Obes. Surg. 2009;19:139-145.

7. White MA, Kalarchian MA, Masheb RM, Marcus MD, Grilo CM. Loss of control over eating predicts outcomes in bariatric surgery patients: a prospective, 24 month follow up study. J. Clin. Psychiatry 2010; 71: 175-184.

8. Burgmer R, Grigutsch K, Zipfel S, et al. The influence of eating behavior and eating pathology on weight loss after gastric restriction operations. Obes. Surg. 2005; 15: 684-691. 